\title{
Comparaçáo da eficiência de diferentes armadilhas utilizadas para a captura de Dermanyssus gallinae (Acari: Dermanyssidae) (de Geer, 1778)
}

\author{
Comparison of different sampling traps for Dermanyssus gallinae
}

(Acari: Dermanyssidae) (de Geer, 1778)

Lucas M. Cunha ${ }^{1}$; Mariana M. Cunha²; Romário C. Leite ${ }^{3}$; Israel J. Silva ${ }^{3}$; Paulo R. de Oliveira ${ }^{3 *}$

${ }^{1}$ Doutorando em Ciência Animal (Epidemiologia), Escola de Veterinária, Universidade Federal de Minas Gerais - UFMG

${ }^{2}$ Graduação em Ciências Biológicas, Instituto de Ciências Biológicas, Universidade Federal de Minas Gerais - UFMG

${ }^{3}$ Departamento de Medicina Veterinária Preventiva, Escola de Veterinária, Universidade Federal de Minas Gerais - UFMG

Recebido em 19 de Janeiro de 2009

Aceito em 19 de Maio de 2009

\section{Resumo}

O presente trabalho teve como objetivo comparar a eficiência de armadilhas de papel corrugado com a de armadilhas de colmo de bambu taquaril (Phyllostachys sp.) seco na captura de Dermanyssus gallinae, presentes em uma bateria de gaiolas de metal utilizadas na criaçáo de galinhas de postura. A presença de ovos no interior de cada tipo de armadilha foi comparada por meio do teste de Qui-Quadrado e pelo intervalo de confiança para duas proporçóes. Os valores das somas de estágios móveis nas diferentes armadilhas a cada intervalo de tempo foram comparados utilizando-se o teste de Wilcoxon. A proporçáo de armadilhas contendo ovos não foi diferente nos dois tipos de armadilha $(\mathrm{p}<0,05)$. $\mathrm{O}$ número total de estágios móveis capturados durante a coleta a cada dois dias de intervalo em cada tipo de armadilha foi diferente ( $\mathrm{p} \leq 0,01)$, enquanto que as contagens, nas armadilhas de papelão, apresentaram distribuição normal e foram superiores aos valores de espécimes móveis capturados nas armadilhas de bambu.

Palavras-chave: Ácaros hematófagos, Dermanyssus gallinae, armadilha.

\begin{abstract}
This work aims to compare the performance of corrugated paper and "taquaril" bamboo (Phyllostachys sp.) straw traps for collecting (in sampling) Dermanyssus gallinae in a metal cages battery laying hens. The presence of eggs in the two trap models were compared using a Qui-square test and a proportion confidence interval test. Total daily values of mobile instars gathered in each type of trap were compared using the Wilcoxon's test. The amount of traps containing eggs was not different in neither of the traps $(\mathrm{p}<0,05)$. The number of mobile instars sampled at every two days per trap model was different $(\mathrm{p} \leq 0,01)$. Counting in the corrugated paper traps showed a Gauss distribution histogram and was superior to the values of the bamboo straw traps.
\end{abstract}

Keywords: Poultry red mite, Dermanyssus gallinae, sampling trap.

\section{Introdução}

Dermanyssus gallinae é um ácaro hematófago, popularmente denominado "ácaro vermelho das galinhas" ou "micuim". É também erroneamente chamado de "piolho de galinha" ou "piolho dos ninhos". Pertence à ordem Mesostigmata e à família Dermanyssidae. Representa, no Brasil e no mundo, um sério problema sanitário para a avicultura comercial de postura.

\footnotetext{
*Autor para correspondência: Paulo R. de Oliveira

Professor Associado, Departamento de Medicina Veterinária Preventiva, Escola de Veterinária, Universidade Federal de Minas Gerais - UFMG, Av. Antônio Carlos 6627, CP 567, CEP 30123-970, Campus da UFMG, Belo Horizonte - MG, Brasil;

e-mail: pro@ufmg.br
}

Um levantamento em aviários industriais de postura do Estado de São Paulo constatou que 25,6\% das granjas apresentavam Dermanyssus gallinae, 13,9\% apresentavam Ornithonyssus sylviarum (CANESTRINI; FANZAGO, 1877) e 34,9\% apresentavam ambas as espécies (TUCCI et al., 1996). D. gallinae determina grandes prejuízos aos criadores, principalmente devido à reduçáo na produção de ovos e redução na taxa de ganho de peso de galinhas em crescimento (GUIMARÂES; TUCCI; BARROS-BATTESTI, 2001; KILPINEN et al., 2005). O parasitismo por essa espécie de ácaro causa anemia em galinhas, e diversos autores demonstram a relação dessa espécie de ácaro com diferentes microrganismos patogênicos (KIRKWOOD, 1967; VALIENTE-MORO; 
CHAUVE; ZENNER, 2007; SMITH; BLATTNER; HEYS, 1944). $\mathrm{O}$ ataque em humanos também é frequentemente relatado, provocando dermatites (AUGER et al., 1979; ROSEN; YEUHAM; BRAVERMAN, 2002).

Ninfas e adultos de D. gallinae alimentam-se melhor à noite ou em ambiente escuro, mas podem alimentar-se durante o dia sob condições de baixa iluminação. Se criado isolado em câmaras, esse ácaro adquire a necessidade de se alimentar após poucos dias. Trata-se de uma espécie que se ingurgita completamente após um único repasto sanguíneo e, sob esse aspecto, difere de Ornithonyssus bursa (BERLESE, 1888) e O. sylviarum. De forma semelhante a percevejos, $D$. gallinae forma grupos de ácaros reunidos em frestas encontradas, por exemplo, nos comedouros e bebedouros das aves (SIKES; CHAMBERLAIN, 1954). No Brasil, aspectos da biologia de D. gallinae em condiçóes de laboratório demonstraram que seu ciclo de desenvolvimento, assim como o de grande parte dos Mesostigmata parasitos, é bastante curto, com duração de sete a oito dias (TUCCI; GUIMARÁES, 1998). Adultos são capazes de sobreviver 34 semanas sem realizar repasto sanguíneo (KIRKWOOD, 1963).

A utilizaçấo de diferentes tipos de armadilhas pode ser empregada para o monitoramento da dinâmica populacional (NORDENFORS; HÖGLUND, 2000), para a aplicação de acaricidas nas granjas (NORDENFORS; HÖGLUND, 2000; LUNDH; WIKTELIU; CHIRICO, 2005) e para a avaliação da resposta a medidas de controle dessa espécie de ácaro (NORDENFORS; CHIRICO, 2001; MEYER-KÜHLING et al., 2007). Portanto, diferenças de desempenho e função de diferentes tipos de armadilha precisam ser avaliadas para a escolha de armadilhas adequadas a diferentes situaçóes. O presente trabalho teve como objetivo verificar e comparar a presença de ovos e o número médio de espécimes móveis de $D$. gallinae, capturados nas armadilhas de papel corrugado e de colmo de bambu taquaril (Phyllostachys sp.).

\section{Material e Métodos}

Um escape de $D$. gallinae ocorreu em uma sala utilizada para a criação de ectoparasitos de aves na Escola de Veterinária da UFMG. Estes ácaros colonizaram uma bateria de gaiolas de metal utilizadas na criação de galinhas usadas para alimentação de Argas (Persicargas) miniatus (KOCH, 1844). Aproveitando esse incidente, foi realizado um estudo para se comparar a eficiência de armadilhas de papeláo e de bambu na captura de D. gallinae. Esses bioensaios foram realizados no período de 17 de fevereiro a 04 de março de 2007, quando a temperatura no local oscilou entre 20,9 e $26,9^{\circ} \mathrm{C}$, e a umidade relativa do ar entre 54 e $86 \%$.

Neste estudo, as três gaiolas utilizadas para a criação de galinhas eram de arame $(35 \mathrm{~cm}$ de largura, $40 \mathrm{~cm}$ de comprimento e $50 \mathrm{~cm}$ de altura), sem contato com o chão, apoiadas em suportes presos às paredes da sala, com comedouros e um bebedouro em calha, todos em metal. Para facilitar o manejo das galinhas, a calha de fornecimento de água foi acoplada a uma garrafa de polietileno (PET) de $2 \mathrm{~L}$, que funcionava como reservatório. A calha, a garrafa e o comedouro foram afixados às gaiolas. Para evitar a fuga dos ácaros para o restante da sala, uma camada de detergente comercial (alquil benzeno sulfonato de sódio linear) foi aplicada nas paredes e piso. A limpeza do ambiente era feita a cada dois dias.

Cada gaiola recebeu uma galinha adulta. As aves das extremidades eram da raça Leghorn branca, em fase de postura e, a da gaiola central, da raça Garnisé. A presença de colônias de D. gallinae foi assegurada pela observação de ácaros avermelhados nos pontos de encontro de fios de arame da gaiola, frestas do bebedouro e do comedouro e, principalmente, nos barbantes.

Foram comparados dois tipos de armadilha para a captura de D. gallinae. O primeiro consistiu em pedaços de papel corrugado (papeláo) com $100 \mathrm{~mm}$ de largura, $70 \mathrm{~mm}$ de comprimento e $3 \mathrm{~mm}$ de altura. O segundo tipo constituiu-se de um colmo seco de bambu taquaril (Phyllostachys sp.), partido ao meio em seu sentido longitudinal, amarrado com cordôes de algodáo e aquecido em estufa a $100^{\circ} \mathrm{C}$ por duas horas, para eliminar possíveis artrópodes contaminantes. Em uma das extremidades de uma das metades, foi feito um corte de 10-20 × 1-2 mm para permitir a entrada dos ácaros.

Quatro pontos no vértice superior da gaiola central foram selecionados como pontos de colocaçáo das armadilhas. Duas armadilhas de papelâo e duas de bambu foram amarradas com cordáo nos diferentes pontos selecionados. A posiçáo de cada armadilha foi selecionada aleatoriamente por sorteio, com o objetivo de náo expor um determinado tipo de armadilha a pontos com maior infestação (próximos dos cordóes, utilizados para fixar as garrafas PET, por exemplo). A troca das armadilhas foi feita a cada dois dias, quando entấo foram colocadas em sacos plásticos. Estes eram lacrados e armazenados em um freezer $\left(-18 \mathrm{a}-22^{\circ} \mathrm{C}\right)$ por 20 a 24 horas. Após o congelamento, as armadilhas foram abertas sobre uma bandeja branca, e os ácaros contados por meio de sucção por uma pipeta do tipo Pasteur, acoplada a uma bomba de vácuo $\left(\mathrm{FANEM}^{\circledast}, 089 \mathrm{CAL}\right)$ por meio de uma mangueira de silicone.

Foram distribuídas 36 armadilhas ao longo do experimento, divididas em grupos de quatro armadilhas a cada dois dias, sendo duas de cada tipo. Para as contagens de estágios móveis (larvas, ninfas e adultos), optou-se por considerar as médias de cada tipo de armadilha a cada dia como uma repetiçáo, totalizando, portanto, nove repetiçôes por tipo de armadilha. Esse procedimento foi empregado com o objetivo de evitar variaçóes na contagem devido à posição da armadilha na gaiola, escolhida aleatoriamente.

A presença de ovos no interior de cada tipo de armadilha foi comparada por meio do teste Qui-Quadrado e pelo valor do intervalo de confiança para duas proporçóes, segundo Nogueira et al. (1997). A cada dia de contagem, os valores das somas dos estágios móveis, nas diferentes armadilhas, foram comparados utilizando-se o teste de Wilcoxon, segundo Sampaio (2002).

\section{Resultados e Discussão}

Ambas as armadilhas de papel corrugado e de bambu taquaril foram eficientes como abrigos e para a postura de D. gallinae. Em apenas duas repetiçôes de cada tipo de armadilha não foram observados ovos de D. gallinae. A proporçáo de armadilhas contendo ovos foi igual nos dois tipos de armadilha (Tabela 1). Esse fato revela que ambas as armadilhas funcionam como um abrigo 
Tabela 1. Número mediano de estágios móveis (larvas, ninfas e adultos) coletados em diferentes tipos de armadilha e proporçáo de armadilhas com ovos.

\begin{tabular}{ccc}
\hline Tipo de armadilha & Papel corrugado & Colmo de bambu \\
\hline $\begin{array}{c}\text { número mediano de } \\
\text { estágios móveis }\end{array}$ & $2211^{\mathrm{c}}$ & $916^{\mathrm{d}}$ \\
$\begin{array}{c}\text { proporção de armadilhas } \\
\text { com ovos }\end{array}$ & $87,5 \% \mathrm{o}^{\mathrm{e}}$ & $87,5 \%^{\mathrm{e}}$ \\
\hline
\end{tabular}

${ }^{a}$ Letras sobrescritas diferentes nesta linha diferem estatisticamente pelo teste de Wilcoxon ( $\mathrm{p} \leq 0,01)$. ${ }^{\mathrm{b}}$ Letras sobrescritas iguais não diferem estatisticamente pelo teste de Qui-Quadrado e pelo intervalo de confiança para duas proporçôes $(\mathrm{p}<0,05)$.

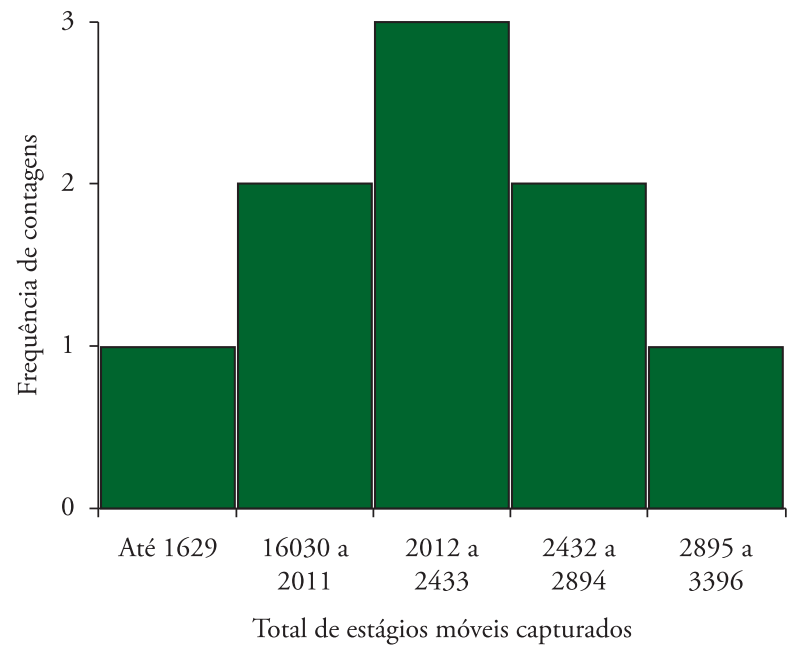

Figura 1. Histograma das contagens de estágios móveis de Dermanyssus gallinae em armadilhas de papel corrugado.

para que as fêmeas desse ácaro realizem oviposição. Entretanto, deve-se ressaltar que é mais fácil retirar espécimes (móveis ou ovos) das armadilhas de bambu que do papel corrugado. Novos testes devem ser feitos para verificar a hipótese de substituição dos abrigos de papel corrugado nos métodos de criação de gamasídeos (Mesostigmata), por alimentação in vivo em laboratório, sugeridos por Chamberlain e Sikes (1950); Camin e Ehrlich (1960) e Durden e Linthicum (1992).

O número de estágios móveis capturados na coleta a cada dois dias em cada tipo de armadilha foi diferente. As contagens de espécimes móveis nas armadilhas de papelão foram superiores ao número encontrado nas armadilhas de bambu (Tabela 1). Em todos os dias, o número médio de ácaros coletados nas armadilhas de bambu foi inferior ao obtido nas armadilhas de papel corrugado. Portanto, para estudos rápidos de detecção de D. gallinae em granjas avícolas, as armadilhas de papeláo do tipo descrito por Nordenfors e Chirico (2001), com dimensóes de $100 \mathrm{~mm}$ de largura, $70 \mathrm{~mm}$ de comprimento e $3 \mathrm{~mm}$ de altura, são mais indicadas que armadilhas de colmo de bambu.

O histograma das raízes quadradas das contagens, obtidas nas armadilhas de papel corrugado (Figura 1), confirma que essa variável tem distribuiçáo normal, conforme resultados obtidos

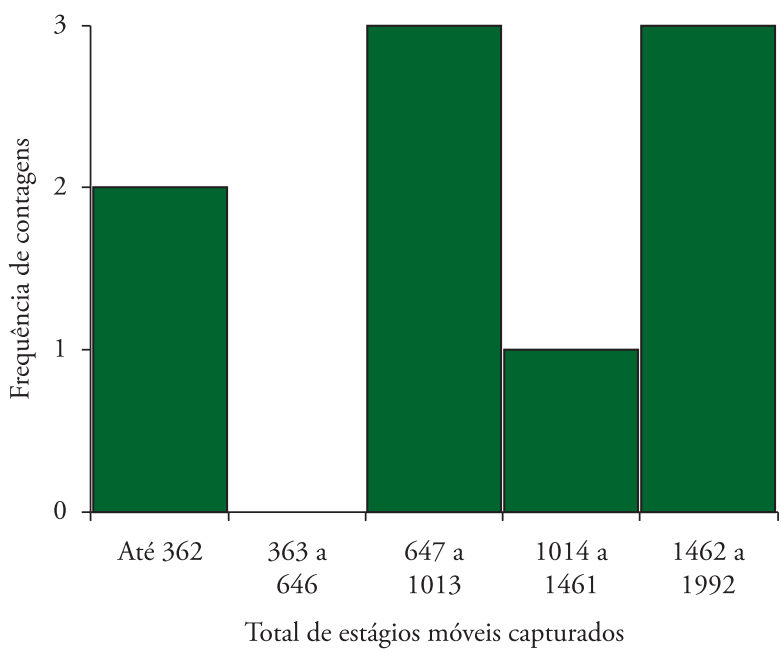

Figura 2. Histograma das contagens de estágios móveis de Dermanyssus gallinae em armadilhas de colmos de bambu taquaril.

por Nordenfors e Chirico (2001). As contagens nas armadilhas de bambu revelam uma grande variabilidade de resposta dessa armadilha, possivelmente devido às diferentes dimensões de cada colmo (Figura 2). As armadilhas de bambu não estavam repletas de ácaros e, nas armadilhas em que esse fato ocorreu, as contagens superaram aquelas encontradas nas armadilhas de papelão. Como o tamanho de armadilhas de papelão e o intervalo de sua retirada do ambiente influenciam nas contagens de ácaros, novos estudos precisam ser feitos para se avaliar o efeito do intervalo de exposição de armadilhas de bambu na contagem de ácaros.

\section{Agradecimentos}

Ao Conselho de Desenvolvimento Científico e Tecnológico (CNPq), pelo apoio financeiro.

Ao Setor de Doenças das Aves da Escola de Veterinária da UFMG, pela doação das aves e gaiolas utilizadas no experimento.

\section{Referências}

AUGER, P. et al. Skin acariasis caused by Dermanyssus gallinae (de Geer): an in-hospital outbreak. Canadian Medical Association Journal, v. 120, n. 6, p. 700-703, 1979.

CAMIN, J. H.; EHRLICH, P. R. A cage for maintaining stock colonies of parasitic mites and their hosts. The Journal of Parasitology, v. 46, n. 1, p. 109-111, 1960.

CHAMBERLAIN, R. W.; SIKES, R. K. Laboratory rearing methods for three common species of bird mites. The Journal of Parasitology, v. 36, n. 5, p. 461-465, 1950.

DURDEN, L. A.; LINTHICUM, K. J. Efficient Laboratory Maintenance of Blood-Feeding Mesostigmatid Mites (Acari). Journal of Medical Entomology, v. 29, n. 3, p. 564-566, 1992. 
GUIMARÃES, J. H.; TUCCI, E. C.; BARROS-BATTESTI, D. M. Ectoparasitos de Importância Veterinária. São Paulo: Plêiade/FAPESP, 2001. 218 p.

LUNDH, J.; WIKTELIU, D.; CHIRICO, J. Azadirachtin-impregnated traps for the control of Dermanyssus gallinae. Veterinary Parasitology, v. 130, n. 3-4, p. 337-342, 2005.

KILPINEN, O. et al. Influence of Dermanyssus gallinae and Ascaridia galli infections on behavior and health of laying hens (Gallus gallus domesticus). British Poultry Science, v. 46, n. 1, p. 26-34, 2005.

KIRKWOOD, A. C. Anaemia of poultry infested with the red mite Dermanyssus gallinae. The Veterinary Record, v. 80, n. 17, p. 514-516, 1967.

KIRKWOOD, A. C. Longevity of the mites Dermanyssus gallinae and Liponyssus sylviarum. Experimental Parasitology, v. 14, n. 3, p. 358-366, 1963.

MEYER-KÜHLING, B. et al. Field efficacy of phoxim 50\% (ByeMite") against the poultry red mite Dermanyssus gallinae in battery cages stocked with laying hens. Veterinary Parasitology, v. 147, n. 3-4, p. 289-296, 2007.

NOGUEIRA, M. L. G. et al. Introduçáo à Bioestatística. Belo Horizonte: Departamento de Estatística do Instituto de Ciências Exatas, Universidade Federal de Minas Gerais, 1997. 215 p.

NORDENFORS, H.; CHIRICO, J. Evaluation of a Sampling Trap for Dermanyssus gallinae (Acari: Dermanyssidae). Journal of Economic Entomology, v. 94, n. 6, p. 1617-1621, 2001.
NORDENFORS, H.; HÖGLUND, J. Long term dynamics of Dermanyssus gallinae in relation to mite control measures in aviary systems for layers. British Poultry Science, v. 41, n. 5, p. 533-540, 2000.

ROSEN, S.; YERUHAM, I.; BRAVERMAN, Y. Dermatitis in humans associated with the mites Pyemotes tritici, Dermanyssus gallinae, Ornithonyssus bacoti and Androlaelaps casalis in Israel. Medical and Veterinary Entomology, v. 16, n. 4, p. 442-444, 2002.

SAMPAIO, I. B. M. Estatística aplicada à experimentaçáo animal. Belo Horizonte: FEPMVZ-Editora, 2002. 265 p.

SIKES, R. K.; CHAMBERLAIN, R. W. Laboratory observations on three species of bird mites. The Journal of Parasitology, v. 40, n. 6, p. 691-697, 1954.

SMITH, M. G.; BLATTNER, R. J.; HEYS, F. M. The isolation of the St. Louis encephalitis virus from chicken mites (Dermanyssus gallinae) in nature. Science, v. 100, n. 2599, p. 362-363, 1944.

TUCCI, E. C. et al. Ocorrência de ácaros hematófagos em aviários de postura no Estado de São Paulo. Revista Brasileira de Parasitologia Veterinária, v. 5, n. 2, p. 95-102, 1996.

TUCCI, E. C.; GUIMARÁES, J. H. Biologia de Dermanyssus gallinae (De Geer, 1778) (Acari, Dermanyssidae). Revista Brasileira de Parasitologia Veterinária, v. 7, n. 1, p. 27-30, 1998.

VALIENTE-MORO, C.; CHAUVE, C.; ZENNER, L. Experimental infection of Salmonella Enteritidis by the poultry red mite, Dermanyssus gallinae. Veterinary Parasitology, v. 146, n. 3-4, p. 329-336, 2007. 